\title{
P-89
}

\section{The Study of Caffeine as Novel Quorum Sensing Inhibitor}

\author{
Siti Nur Maisarah Norizan ${ }^{*}$, Kok- Gan Chan and Wai-Fong Yin
}

Di vision of Genetics and Molecular Biology, Institute of Biological Sciences, Faculty of Science, University of Malaya, Kuala Lumpur 50603, Malaysia; E-mail: outfeet@yahoo.com

Quorum sensing enables bacterial to communicate and at the same time control the gene expression in response to the cell density [1]. It is widely used by both gram-positive and gram-negative bacteria to regulate variety of bacterial physiological functions such as biofilm formation [2], bioluminescence [3], virulence factors [4] and swarming [5] which has been shown contribute to bacterial pathogenesis. With this knowledge, the use of QS inhibitor would be a particular value in treating bacterial pathogenicity and infections. In this work we have tested caffeine as quorum sensing inhibitor by using C.violaceum CV026 as a bioassay. This C.violaceum CV026 mutant strain is incapable of producing the purple pigment called violacein unless there are exogenous supply of $N$-hexanoylhomoserine lactone (C6-HSL) or other short chain AHL .The Inhibitory activity was measured by quantifying violacein production using a spectrophotometer. The results have revealed that Caffeine significantly reduced violacein production in a concentration dependent manner, indicating inhibition of quorum sensing. The presence of caffeine that exhibit anti-quorum sensing activity may be useful as the lead of anti-infective drugs.

\section{REFERENCES}

[1] Schauder S, Bassler BL. The languages of bacteria. Genes Dev 2001; 15: 1468-80.

[2] Merritt J, Qi, F, Goodman SD, Anderson MH, Shi W. Mutation of luxS affects biofilm formation in Streptococcus mutans. Infect Immun 2003; 71: 1972-9.

[3] Miller MB, Bassler BL. Quorum sensing in bacteria. Annu Rev Microbiol 2001; 55: 165-99.

[4] Mellbye B, Schuster M. The sociomicrobiology of antivirulence drug resistance: A proof of concept. mBio 2011; 2: e00131-11.

[5] Tremblay J, Richardson AP, Lépine F, Déziel E. Self-produced extracellular stimuli modulate the Pseudomonas aeruginosa swarming motility behaviour. Environ Microbiol 2007; 9: 2622-30. 